\title{
Amontons' Rules of Friction Formulated 300 Years Ago
}

The Egyptians did it with sand and water; the Greeks and Italians, with olive oil; medieval Europeans used pebbles and soft metals. All found tricks to start and keep moving skids, pulleys, and grinding mills. But before the 17 th century, few took the time to scientifically study frictional forces in machinery. It was the deaf French physicist Guillaume Amontons (1663-1705) who, in 1699, reported results of simple experiments and formulated rules about friction that could be tested and applied by the engineer in the emerging age of machinery. Three hundred years after this seminal publication "De la Resistance Causée dans les Machines" (in Mem. de l'Académie Royale A [1699], p. 275), tribologists remember Amontons as the father of modern studies of friction.

In his paper, Amontons motivated the studies by referring to the importance of machines, but decrying the lack of attention paid to effects of friction. His apparatus is not unfamiliar: leaf springs for loading the substrate and coiled springs for measuring tangential resistance. Iron, copper, lead, and wood were the materials tested, and later he mentioned that they were "spread" with "old fat." (Hence, what is frequently referred to as Amontons' laws of "dry-sliding" friction should actually be called laws of "boundary lubrication.") The main findings were that the resistance caused by friction is proportional to the load and independent of the extent of contact of the rubbing parts, that is, independent of the apparent area of contact. These findings are now known as Amontons' first and second laws of friction. He also reported that the ratio of (tangential) resistance to load was about $1 / 3$ for most combinations of "greased" materials, in effect defining what we now call the coefficient of friction and assigning it a constant value. Amontons also speculated about the fundamental nature of friction and interpreted the observed behavior geometrically. Resistance to tangential motion was caused by irregularities (asperities) on both surfaces riding over each other. $\mathrm{He}$ also recognized that both rigid and elastic asperities gave the same resistance; the former via lift, the latter via elastic deformation. Finally, like any good engineer, Amontons showed how friction contributes to various components in machinery like ropes and pulleys, provided rules, presented sample calculations, then gave lookup tables for engineers to avoid having to do the calculations.

Amontons' solution to reducing friction in machinery was not new. Ancient
Egyptians poured water on wooden planks to move large stone statues and lubricated the axles of their chariots with animal tallow. Pliney the Elder and Cato advised ancient Romans that olive oil dregs lubricated better than animal fat (the former did not solidify as fast as the latter). Artisans in the middle ages used natural solids like stones and woods as well as processed metals such as carbonized steels and bronzes to overcome static friction and reduce kinetic friction in wheels and shafts; scarcity of such friction reducing materials made tribologists of many an artisan. Early versions of retainer bearings and spline shafts and gears appeared in wood cut prints in the 11th-13th centuries.

Even more startling were Leonardo da Vinci's ingenious bearing designs, many hidden from the public until the rediscovery of his Madrid codices (ca. 1495) in 1967. Not surprisingly, da Vinci also studied friction. In the codices, he sketched inclined planes used to measure friction of blocks dragged along various faces. Like Amontons, da Vinci observed that friction was proportional to load and independent of apparent contact area. He, too, deduced that the coefficient of friction was a meaningful concept and suggested that most materials give a friction coefficient of about $1 / 4$, not far from the $1 / 3$ value suggested by Amontons 200 years later.

Why then shouldn't we consider da $V$ inci as the father of modern friction? For one thing, da Vinci was more concerned with imaginative ideas and elegant theories than with practical solutions and verification of hypotheses. Otherwise, how could this brilliant painter, familiar with most of the oils used to make paints, not have evaluated them as antifriction agents? For another, he did not get his results out to fellow tribologists for peer review-there were no scientific societies or journals to diffuse his Renaissance wisdom to the engineering community.

Unlike da Vinci, Amontons was a product of contemporary scientific culture. Early in the 17th century, Roger Bacon prescribed that experimentation, not elegance, be the basis of scientific theory. By 1687 , Newton's Principia established the language of mechanics, as well as postulating viscous flow, the basis of modern fluidfilm lubrication. Forums for open discussion of scientific investigation were started in France (the Académie Royale des Sciences) and in England (the Royal Society). These societies invited natural philosophers to present, challenge, and expand upon current knowledge. Indeed
Amontons' 1699 paper on friction was controversial. The president of the French Académie was motivated to verify Amontons' results, which he did, adding his own speculations on causes of frictional resistance.

And so it went, over the next several hundred years. Within five years of Amontons' paper, his laws were verified twice and introduced into engineering practice. In addition, new concepts like deformation and shear of asperities were added to the growing list of mechanisms contributing to frictional resistance. In the 18 th century, scientists like Coulomb, Leibnitz, and Euler tackled quantification of friction processes while others postulated mechanisms of adhesion and deformation, a debate that rages on into the 20th century. With current atomistic understanding of materials and more sensitive surface probes, new insights into friction processes abound (see MRS Bulletin, June 1998). Yet with all this sophistication, engineers still make use of Amontons' laws to solve practical problems.

How should we scientists pay homage in this, the Amontons' tricentennial year? Amontons personally does not need the recognition; he is already known as the inventor of the modern telegraph and as the father of modern-day thermometry for discovering that the pressure of a fixed quantity of gas is proportional to the temperature (the "other" Amontons' Law). He even has a crater on the moon named after him! Perhaps we should admire those unknown resourceful and desperate artisans who, like modern engineers, discovered and rediscovered solid and liquid lubricants $\longrightarrow$ often by serendipity-to mobilize the ancient world? Or perhaps we should be inspired by our modern scientific ancestors who taught us that coherent transmission of knowledge makes serendipitous discoveries more likely? One thing's for sure: We owe Amontons and his 17th century colleagues a debt of gratitude for ushering in an open scientific culture.

\section{Naval Research Laboratory}

For FurTHER READING: Duncan Dowson, History of Tribology, 1st ed (Longman, London, 1979), 2d ed (Professional Engineering Publishing, London, 1998); F.P. Bowden and D. Tabor, The Friction and Lubrication of Solids, Part II, Chap. 24 (Oxford University Press, London, 1964); and John Muendel, "Friction and Lubrication in Medieval Europe," ISIS 86 (1995) p. 373. 